\title{
A Projection Argument for Differential Inclusions, with Applications to Persistence of Mass-Action Kinetics
}

\author{
Manoj GOPALKRISHNAN ${ }^{\dagger}$, Ezra MILLER $\ddagger$ and Anne SHIU ${ }^{\S}$ \\ $\dagger$ School of Technology and Computer Science, Tata Institute of Fundamental Research, \\ 1 Homi Bhabha Road, Mumbai 400 005, India \\ E-mail: manojg@tifr.res.in \\ URL: http://www.tcs.tifr.res.in/ manoj/ \\ $\ddagger$ Department of Mathematics, Duke University, Box 90320, Durham, NC 27708-0320, USA \\ E-mail: ezra@math.duke.edu \\ URL: http://www.math.duke.edu/ ezra/ \\ $\S$ Department of Mathematics, University of Chicago, \\ 5734 S. University Avenue, Chicago, IL 60637, USA \\ E-mail: annejls@math.uchicago.edu \\ URL: http://math.uchicago.edu/ annejls/
}

Received August 07, 2012, in final form March 23, 2013; Published online March 26, 2013

http://dx.doi.org/10.3842/SIGMA.2013.025

\begin{abstract}
Motivated by questions in mass-action kinetics, we introduce the notion of vertexical family of differential inclusions. Defined on open hypercubes, these families are characterized by particular good behavior under projection maps. The motivating examples are certain families of reaction networks - including reversible, weakly reversible, endotactic, and strongly endotactic reaction networks - that give rise to vertexical families of massaction differential inclusions. We prove that vertexical families are amenable to structural induction. Consequently, a trajectory of a vertexical family approaches the boundary if and only if either the trajectory approaches a vertex of the hypercube, or a trajectory in a lower-dimensional member of the family approaches the boundary. With this technology, we make progress on the global attractor conjecture, a central open problem concerning mass-action kinetics systems. Additionally, we phrase mass-action kinetics as a functor on reaction networks with variable rates.
\end{abstract}

Key words: differential inclusion; mass-action kinetics; reaction network; persistence; global attractor conjecture

2010 Mathematics Subject Classification: 34A60; 80A30; 92C45; 37B25; 34D23; 37C10; 37C15; 92E20; 92C42; 54B30; 18B30

\section{Introduction}

The global attractor conjecture has been a central open problem in reaction network theory since its formulation by Horn in 1974 [16]. It asserts that any complex-balanced mass-action kinetics system of ordinary differential equations with positive initial conditions possesses a globally attracting stationary point in each stoichiometric compatibility class (see Conjecture 6.1 for a more precise statement). It is well-known that this conjecture is implied by Feinberg's persistence conjecture [11, Remark 6.1.E], a version of which asserts the following: for weakly reversible networks taken with mass-action kinetics, no species asymptotically becomes extinct or unbounded.

An a priori special case of the persistence conjecture asserts that at least one species survives asymptotically. In this paper, we show that this a priori special case in fact implies the entire 
persistence conjecture (Corollary 6.3). As a consequence, if the persistence conjecture is false, then in every minimal counterexample each species becomes either extinct or unbounded. It follows that the persistence conjecture in dimension $n$ implies the global attractor conjecture in dimension $n+1$ (Theorems 6.8 and 6.10).

Pantea [20], building on earlier work by Craciun, Nazarov, and Pantea [10], used the persistence conjecture in two dimensions to prove the global attractor conjecture in three dimensions. Pantea's work relied in part on projecting trajectories to lower-dimensional faces. Here, we generalize this projection argument in two ways. First, our main result, Theorem 3.15, applies in arbitrary dimensions. Second, our results hold not only for mass-action kinetics networks, but for certain families of differential inclusions that we call vertexical (Definition 3.13). The notion of vertexical family makes precise the essential structure required of a family of dynamical systems on hypercubes of varying dimensions to permit a structural induction argument of this sort. Theorem 3.15 shows that a trajectory in a vertexical family approaches the boundary if and only if either it approaches a vertex of the hypercube, or a lower-dimensional trajectory in the family approaches the boundary.

Vertexical families of differential inclusions arise naturally in reaction network theory by way of mass-action kinetics or, more generally, power-law dynamics that are considered in biochemical systems theory (Remark 4.2), on networks that are reversible, weakly reversible, endotactic, strongly endotactic (Definition 4.6.4), and so on. We prove that these networks, and more generally, projective classes of networks (Definition 5.1), give rise to vertexical families of massaction differential inclusions (Theorem 5.23 and Corollary 5.24).

In the course of proving this result, we are led to view mass-action kinetics as a functor (Theorem 5.20). No more category theory is required in this paper beyond the definition of a functor. Functoriality itself is used as a convenient shorthand for a list of properties spelled out at the beginning of Section 5. The use of this shorthand clarifies the concept of vertexical family and suggests that other questions concerning mass-action kinetics systems may be amenable to structural induction (Question 5.26). Section 6 discusses the implications of our results for persistence of mass-action kinetics systems.

\section{Dynamical properties of differential inclusions}

In this section, we recall certain dynamical properties of differential inclusions defined on manifolds. For background on manifolds, see [19]. All manifolds considered here have finite dimension. For background on differential inclusions, see [7].

Definition 2.1. Let $M$ be a smooth manifold with tangent bundle $\pi_{M}: T M \rightarrow M$. A differential inclusion on $M$ is a subset $X \subseteq T M$.

Example 2.2. The simplest differential inclusions on $M$ are vector fields on $M$. The subset $X \subseteq T M$ for a given vector field is the image of the corresponding section $M \hookrightarrow T M$.

Definition 2.3. Fix a differential inclusion $X$ on a smooth manifold $M$. Let $I \subseteq \mathbb{R}_{\geq 0}$ be a nonempty interval (in particular, connected) containing its left endpoint. A differentiable curve $f: I \rightarrow M$ is a trajectory of $X$ if the tangent vectors to the curve lie in $X$. An unbounded interval is a ray. A trajectory defined on a ray eventually has a property $P$ if there exists $T>0$ such that property $P$ holds for the function for all $t \geq T$.

Let $\bar{M}$ be a smooth manifold with corners. That is, $\bar{M}$ is a space locally modeled on the closed nonnegative orthant [19, p. 363]. Then $\partial \bar{M}$ denotes the boundary of $\bar{M}$, which is the set

of points of $\bar{M}$ that are not in the relative interior of $\bar{M}$. The relative interior $\bar{M} \backslash \partial \bar{M}$ of $\bar{M}$ is a smooth manifold [19, p. 386, Examples 14-19]. 
Definition 2.4. Let $\bar{M}$ be a smooth manifold with corners with relative interior $M=\bar{M} \backslash \partial \bar{M}$, and let $V \subseteq \partial \bar{M}$ be a subset of the boundary.

1. A differential inclusion $X \subseteq T M$ is persistent relative to $V$ if the closure in $\bar{M}$ of every trajectory of $X$ is disjoint from the closure $\bar{V}$ of $V$ in $\bar{M}$.

2. A differential inclusion $X \subseteq T M$ is repelled by $V$ if for every open set $O_{1} \subseteq \bar{M}$ with $\bar{V} \subseteq O_{1}$, there exists a smaller open set $O_{2} \subseteq O_{1}$ with $\bar{V} \subseteq O_{2}$ such that for every trajectory $f: I \rightarrow M$ of $X$, if $f(\inf I) \notin O_{1}$ then $f(I) \cap O_{2}$ is empty; in other words, if the trajectory begins outside of $O_{1}$, then the trajectory never enters $O_{2}$.

3. If $\bar{M}$ is compact, then a differential inclusion $X \subseteq T M$ is permanent if it is persistent and there is a compact subset $\Omega \subseteq M$ such that for every ray $I$, every trajectory of $X$ defined on $I$ is eventually contained in $\Omega$.

More generally, a set $\mathcal{X}$ of differential inclusions on $M$ is persistent relative to $V$, repelled by $V$, or permanent if every member $X \in \mathcal{X}$ has the corresponding property.

Definition 2.5. Differential inclusions that are persistent relative to the boundary $\partial \bar{M}$ are simply called persistent and similarly for repelled. A collection of differential inclusions, possibly on a family of different manifolds with corners, is persistent, permanent, or repelled if each differential inclusion in the collection has the respective property.

Remark 2.6. Any differential inclusion repelled by $V$ is also persistent relative to $V$. The converse is false in general because different trajectories starting outside $O_{1}$ could get arbitrarily close to $V$. However, certain extra conditions could guarantee that the original differential inclusion is repelled by $V$. For example, suppose $\bar{M}$ is compact and that the differential inclusion $X$ has a continuous extension $\bar{X}$ to $\bar{M}$. Assume further that every trajectory of $\bar{X}$ starting in $\partial \bar{M}$ but outside $\bar{V}$ has its closure disjoint from $\bar{V}$. If the projection $\bar{X} \rightarrow \bar{M}$ is sufficiently nice we are unsure what conditions to impose, but we have in mind properness - then it should be possible to conclude that $X$ is repelled by $V$.

Remark 2.7. A differential inclusion that is permanent need not be repelled by the boundary of $\bar{M}$. The reason appeared already in Remark 2.6: different trajectories starting outside $O_{1}$ could get arbitrarily close to the boundary (on the way to ending up in $\Omega$ ). Conversely, a differential inclusion repelled by the boundary need not be permanent, even if $\bar{M}$ is compact, because $\mathrm{O}_{2}$ might necessarily be smaller when $O_{1}$ is smaller. That is, trajectories that start closer to the boundary could eventually remain closer to the boundary; see Example 2.8.

Example 2.8. Fix a differential inclusion $X$ on a planar disk $\bar{M}$ whose trajectories form concentric circles about its center. $X$ is repelled by the boundary circle $V=\partial \bar{M}$. Indeed, if $O_{1} \subseteq \bar{M}$ is an open set containing $V$, then the compact set $\bar{M} \backslash O_{1}$ achieves a maximum radius $r$ from the center, so we can take $O_{2}$ to be the set of all points in $\bar{M}$ of radius $>r$.

Lemma 2.9. If the differential inclusion $X$ in Definition 2.4 is persistent, then for every trajectory $f: I \rightarrow M$ of $X$, there exist disjoint open sets $O_{f}$ and $O_{V}$ in $\bar{M}$ containing the closures in $\bar{M}$ of $f(I)$ and $V$ respectively.

Proof. A manifold with corners is metrizable, and hence it is a normal Hausdorff space.

Remark 2.10. Consider a differential inclusion defined on a positive orthant $M=\mathbb{R}_{>0}^{n}$. Distinct partial compactifications $\bar{M}$ of $M$ yield distinct notions of persistence. In the case of $\bar{M}=\mathbb{R}_{\geq 0}^{n}$, even with Lemma 2.9, our definition of persistence is weaker than the standard definition [14] that requires each coordinate of a trajectory to remain bounded away from 0 for all time. However, the mass-action differential inclusions that we consider are viewed in the 
compactification $\bar{M}=[0, \infty]^{S}$ (Remark 5.18), so in the context of reaction network theory, our definition of persistence is in fact stronger than the standard definition because coordinates of trajectories not only must be bounded away from zero but also must avoid going to infinity.

Mathematically, the definition we adopt has the advantage of being purely topological, so it behaves well under homeomorphism. Our definition also allows, if required, to separate the usual concept of persistence into the two questions of whether trajectories are bounded and whether $\omega$ limit points exist on the boundary. Both properties are conjectured to hold for weakly reversible and, more generally, endotactic (see Definition 4.6) reaction networks. When trajectories are bounded, our definition of persistence is equivalent to the standard one. Indeed, the mass-action differential inclusions that we introduce later are viewed in the compactification $[0, \infty]^{S}$, so our definition of persistence automatically implies boundedness of trajectories; see Remark 5.18.

Remark 2.11. Suppose a manifold $M$ has a metric d, and $V$ is a compact subset of $M$. A differential inclusion $X$ is repelled by $V$ if for every $d_{1} \in \mathbb{R}_{>0}$, there exists $d_{2} \in \mathbb{R}_{>0}$ such that every trajectory $f: I \rightarrow M$ of $X$ starting with $\mathrm{d}(f(\inf I), V) \geq d_{1}$ maintains $\mathrm{d}(f(I), V) \geq d_{2}$.

Remark 2.12. Our notion of "repelled" is new, motivated by the requirements of Theorem 3.15. The motivations are further explained in Remark 3.24. Cognate but distinct concepts bearing similar names have been defined by others. Anderson and Shiu define a boundary face to have a "repelling neighborhood" if there is a neighborhood of the face such that whenever a trajectory enters that neighborhood, it can get no closer to the face while remaining in that neighborhood [4]. Banaji and Mierczynski define a "repelling face" as a boundary face for which any trajectory that begins in that face immediately exits the face into the interior of the relevant invariant set [8]. Neither of these concepts is adequate for our purposes.

Remark 2.13. Suppose a differential inclusion $X$ is a subset of a persistent differential inclusion $Y$. Then $X$ must be persistent, since each of its trajectories is a trajectory of $Y$. More generally, consider properties $P$ of differential inclusions $X$ of the form " $P(f)$ holds for all trajectories $f$ of $X$." If property $P$ is true for a differential inclusion $Y$, and a differential inclusion $Z$ factors through $Y$, then property $P$ is true for $Z$ as well.

\section{Vertexical families of differential inclusions}

A mass-action kinetics system is naturally defined on the positive orthant $\mathbb{R}_{>0}^{S}$ corresponding to the space of concentrations of species. In this section we work instead with open hypercubes $(0,1)^{S}$, and not directly with positive orthants themselves.

To justify this choice, first we argue that nothing is lost by working with hypercubes. Open hypercubes $(0,1)^{S}$ are diffeomorphic to positive orthants $\mathbb{R}_{>0}^{S}$, so differential inclusions can be transferred from one space to the other by fixing a diffeomorphism and using its Jacobian (see Section 5.2). Additionally, properties such as persistence are defined topologically on the tangent bundle - and therefore invariant under diffeomorphism - so they can be analyzed on either space.

An advantage of working with open hypercubes is that they have natural "cubical" compactifications $[0,1]^{S}$ that appear to be optimally relevant in the context of mass-action kinetics. Alternatively, we could have achieved a cubical compactification by considering the hypercubes $[0, \infty]^{S}$. Nevertheless, there is a stylistic advantage to working with the hypercubes $[0,1]^{S}$ : we can treat symmetrically the cases where a species concentration goes to infinity or to zero. This makes some of our definitions more transparent, and the structural induction becomes cleaner.

Definition 3.1. For any finite nonempty set $S$, let $\mathcal{D}_{S}$ be the set of all differential inclusions on the open hypercube $(0,1)^{S}$. Fix a collection $\mathcal{S}$ of finite nonempty sets. If $\mathcal{X}_{S}$ is a set of differential inclusions on $(0,1)^{S}$ for each $S \in \mathcal{S}$, then the collection $\mathcal{X}=\left\{\mathcal{X}_{S} \subseteq \mathcal{D}_{S}\right\}_{S \in \mathcal{S}}$ of sets $\mathcal{X}_{S}$ is a family of differential inclusions on open hypercubes indexed by $\mathcal{S}$. 


\subsection{Definitions concerning hypercubes}

The definition of vertexical families requires some preliminary notation on hypercubes.

Notation 3.2. Let $S$ be a finite nonempty set.

1. For every $i \in S$, let $e_{i} \in \mathbb{R}^{S}$ be the standard basis vector indexed by $i$; that is, $e_{i}: S \rightarrow \mathbb{R}$ sends $i$ to 1 and $S \backslash\{i\}$ to 0 .

2. For each subset $U \subseteq S$ and vertex $x \in\{0,1\}^{S}$ of the hypercube $[0,1]^{S}$, let

$$
F_{U}(x)=\left(x+\operatorname{span}\left\{e_{i} \mid i \in U\right\}\right) \cap[0,1]^{S}
$$

denote the face of the hypercube $[0,1]^{S}$ along $U$ at vertex $x$.

3. For $p=\sum_{i \in S} p_{i} e_{i} \in \mathbb{R}^{S}$, let $|p|=\sqrt{\sum_{i \in S} p_{i}^{2}}$ denote its Euclidean norm.

4. For subsets $P, Q \subseteq \mathbb{R}^{S}$, denote by $\mathrm{d}(P, Q)=\inf \{|p-q| \mid p \in P$ and $q \in Q\}$ the distance between them.

Remark 3.3. Our notation for faces differs from that in related references $[3,4,5,9,22]$. What those works call $F_{U}$ is close to what we call $F_{S \backslash U}(0)$, where 0 denotes the origin. This correspondence is not perfect; the sets $F_{U}$ in the related works are faces of the nonnegative orthant $\mathbb{R}_{\geq 0}^{S}$, whereas here the sets $F_{U}(x)$ denote faces of hypercubes.

In the context of reaction networks, $S$ indexes the set of reacting chemical species; a chemical complex, being a linear combination of these species, is therefore viewed as a vector in $\mathbb{R}^{S}$ (see Definition 4.1), which has preferred basis vectors $e_{i}$ for $i \in S$.

Definition 3.4. Fix a finite nonempty set $S$. For a face $F$ of $[0,1]^{S}$ and a real number $\eta>1 / 2$, the centered shrinking

$$
\eta F=\{x \in F \mid \mathrm{d}(x, \partial F) \geq(1-\eta) / 2\}
$$

of $F$ is the set of points in $F$ whose distance from the boundary $\partial F$ is at least $(1-\eta) / 2$.

Example 3.5. A centered shrinking of the rightmost face of the 3-cube looks as follows,

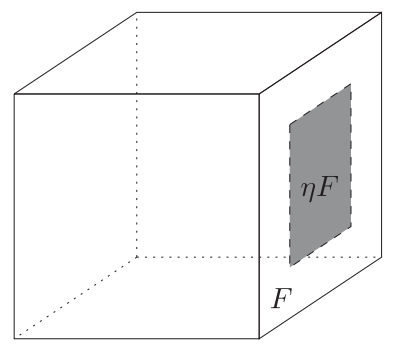

where the inner shaded square is centered in $F$ and has side length $\eta$ times that of $F$.

Definition 3.6. Fix a finite nonempty set $S$ and a subset $U \subseteq S$. Let $P \subseteq[0,1]^{S}$, and suppose that $\varepsilon \in(0,1 / 2) \subseteq \mathbb{R}$. The $\varepsilon$-pile of the subset $P$ along $U$ is the set

$$
\operatorname{pile}(P, \varepsilon, U):=\left\{x+\sum_{i \in U} \varepsilon_{i} e_{i} \mid x \in P \text { and }-\varepsilon \leq \varepsilon_{i} \leq \varepsilon \text { for all } i \in U\right\} \cap[0,1]^{S} \text {. }
$$

Definition 3.7. Fix a finite nonempty set $S$ and a proper face $F$ of the hypercube $[0,1]^{S}$ containing a vertex $x$. Let $U \subseteq S$ be such that $F=F_{S \backslash U}(x)$. For a real number $\varepsilon \in(0,1 / 2)$, the $\varepsilon$-block $F_{\varepsilon}$ is pile $((1-2 \varepsilon) F, \varepsilon, U)$, the $\varepsilon$-pile along $U$ of the centered shrinking $(1-2 \varepsilon) F$. 
Example 3.8. If $\varepsilon=(1-\eta) / 2$ in Example 3.5, the block $F_{\varepsilon}$ looks like the following:

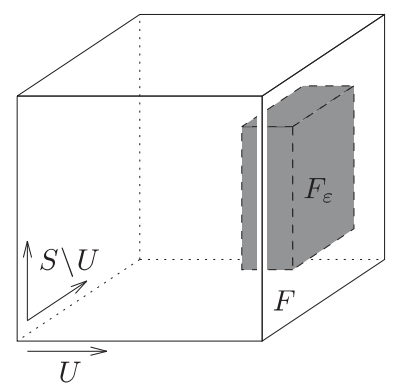

Note that the face $F$ is orthogonal to the basis vector indexed by $U$, and the thickness $\varepsilon$ of the block $F_{\varepsilon}$ equals its distance from the edges of $F$. The vertex $x$ in Definition 3.7 could equally well be any of the four vertices of $F$.

Remark 3.9. The block $F_{\varepsilon}$ is a closed subset of $[0,1]^{S}$. Such sets are closely related to sets that Pantea [20] denoted by $K_{\varepsilon}$, which he used for the purpose of projecting trajectories, as we too do in the current paper.

Notation 3.10. Let $f: U \rightarrow S$ be a map of finite sets, and view $\mathbb{R}^{S}$ as functions $S \rightarrow \mathbb{R}$. Denote by $\pi_{f}: \mathbb{R}^{S} \rightarrow \mathbb{R}^{U}$ the linear projection that sends $v \in \mathbb{R}^{S}$ to $v \circ f \in \mathbb{R}^{U}$. When $U \subseteq S$ and $f$ is inclusion, we write $\pi_{U}$ for the projection map instead of $\pi_{f}$.

Remark 3.11. If $U \subseteq S$ is any subset, then

1) projection is surjective on the open hypercube: $\pi_{U}\left((0,1)^{S}\right)=(0,1)^{U}$, and

2) $\pi_{U}\left((1-2 \varepsilon) F_{S \backslash U}(x)\right)$ is a vertex of the hypercube $[0,1]^{U}$.

Example 3.12. The projection $\pi_{U}$ in Example 3.8 collapses the cube to the horizontal edge

that is $[0,1]=[0,1]^{U}$. The projection takes $F$ as well as the subset $\eta F \subseteq F$ to the indicated right-hand vertex of the interval.

\subsection{Definition of vertexical family and main result}

The heuristic description of a vertexical family of differential inclusions begins by considering a trajectory of a differential inclusion in the family. Suppose the trajectory remains near a face of the hypercube. The vertexical condition requires that, while the trajectory is near the face, the image of the trajectory under the projection map collapsing that face be the trajectory of a fixed lower-dimensional differential inclusion in the family, up to time reparametrization. We emphasize that only the part of the trajectory near the face and away from the boundary of the face is required to be projectable.

Definition 3.13. Let $\mathcal{S}$ be the set of all finite nonempty subsets of the positive integers $\mathbb{Z}_{\geq 1}$. A family $\mathcal{X}=\left\{\mathcal{X}_{S}\right\}_{S \in \mathcal{S}}$ of differential inclusions on open hypercubes indexed by $\mathcal{S}$ is vertexical if for each

- set $S \in \mathcal{S}$,

- differential inclusion $X \subseteq T(0,1)^{S}$ in $\mathcal{X}_{S}$,

- proper nonempty subset $U \subseteq S$, and

- face $F=F_{S \backslash U}(x)$ of $[0,1]^{S}$, 
there is $\varepsilon^{\prime}>0$ such that for every $\varepsilon \in\left(0, \varepsilon^{\prime}\right)$, some differential inclusion $Y \in \mathcal{X}_{U}$ has the property that for every trajectory $f: I \rightarrow[0,1]^{S}$ of $X$ with image in the block $F_{\varepsilon}$, there exist

- a trajectory $g: J \rightarrow[0,1]^{U}$ of $Y$, and

- an order-preserving continuous map $\alpha: I \rightarrow J$

such that $\pi_{U} \circ f=g \circ \alpha$.

Examples of vertexical families of differential inclusions include those arising from reversible, weakly reversible, endotactic, or strongly endotactic chemical reaction networks (Definitions 4.3 and 5.16); this is the content of Corollary 5.24, the goal of Sections 4 and 5. Some nuances in the definition are further discussed in Remark 5.21.

We now give a definition, followed by our main result on abstract vertexical families.

Definition 3.14. Fix a finite set $S$ and an index set $R \subseteq \mathbb{Z}_{\geq 1}$, called the repulsing index set. Embed the hypercube $[0,1]^{R \cap S}$ into the hypercube $[0,1]^{S}$ as the face $[0,1]^{R \cap S} \times\{0\}^{S \backslash R}$. A vertex of $[0,1]^{S}$ is charged if it lies in $[0,1]^{R \cap S}$. A face $F$ of $[0,1]^{S}$ is opposite if $F \cap[0,1]^{R \cap S}$ is empty. The charged set is the set $[0,1]^{R \cap S} \cap \partial[0,1]^{S}$.

In practice, $R$ and $S$ are both subsets of a fixed set, and $R$ need not be finite. If $R$ is empty, then by convention $[0,1]^{R \cap S} \times\{0\}^{S \backslash R}$ is the origin. Thus the origin is always charged. The charged set equals $[0,1]^{R \cap S}$ unless $R \supseteq S$, in which case the charged set is $\partial[0,1]^{S}$.

Theorem 3.15. Fix a vertexical family $\mathcal{X}=\left\{\mathcal{X}_{S}\right\}_{S \in \mathcal{S}}$ on open hypercubes indexed by the set $\mathcal{S}$ of all finite nonempty subsets of the positive integers $\mathbb{Z}_{\geq 1}$, and a repulsing index set $R \subseteq \mathbb{Z}_{\geq 1}$. Assume that for every set $S \in \mathcal{S}$, every differential inclusion $X \in \mathcal{X}_{S}$ is

- persistent relative to the union of all opposite faces of $[0,1]^{S}$ and

- repelled by the charged vertices of its hypercube $[0,1]^{S}$.

Then

1. Every such differential inclusion $X$ is persistent relative to the entire boundary $\partial[0,1]^{S}$ and repelled by the charged set $[0,1]^{R \cap S} \cap \partial[0,1]^{S}$ of its hypercube.

2. Fix $S \in \mathcal{S}$ and $X \in \mathcal{X}_{S}$. If, in addition, $X$ is repelled by the union of all opposite faces of $[0,1]^{S}$, then $X$ is repelled by the boundary $\partial[0,1]^{S}$.

Remark 3.16. The differential inclusion in Theorem 3.15.1 is repelled either by the entire boundary of its hypercube (if $R \supseteq S$ ) or by the proper face $[0,1]^{R \cap S}$.

Proof of Theorem 3.15. Fix $S \in \mathcal{S}$. Let $X \in \mathcal{X}_{S}$. We prove that for every proper, positivedimensional face $F=F_{S \backslash U}(x)$ of $[0,1]^{S}$, the following two claims hold.

A. If $X$ is persistent relative to the boundary $\partial F$ of $F$, then $X$ is persistent relative to $F$.

B. If $F$ is not an opposite face and $X$ is repelled by the boundary $\partial F$ of $F$, then $X$ is repelled by $F$.

The remainder of this proof has two components: we first explain how Claims A and B imply parts 1 and 2 of the theorem, and then we prove the two claims.

To start, consider part 1 of the theorem. The differential inclusion $X$ is persistent relative to all vertices of $[0,1]^{S}$, because each vertex is either charged or an opposite face. Using this fact as a base case, Claim A implies, by induction on the dimension of $F$, that $X$ is persistent relative to every proper face of $[0,1]^{S}$. Since the hypercube $[0,1]^{S}$ has only finitely many faces, $X$ is therefore persistent relative to the entire boundary. For repulsion, all vertices of $[0,1]^{S}$ that lie 
in the charged set are charged vertices (see Remark 3.16), so $X$ is repelled by all such vertices by hypothesis. Using this fact as a base case, Claim B implies, by induction on the dimension of faces $F$ of $[0,1]^{S}$ that are in the charged set (and thus are not opposite faces), that $X$ is repelled by the charged set. Hence, part 1 of the theorem holds for $X$, after we prove the two claims.

As for part 2, now assume that $X$ is repelled by the union of opposite faces of $[0,1]^{S}$. We need that $X$ is repelled by non-opposite faces as well. Each vertex of $[0,1]^{S}$ is either charged or an opposite face, so each vertex repels $X$. Using this fact as a base case, Claim B implies, by induction on the dimension of proper non-opposite faces $F$, that $X$ is repelled by every non-opposite face.

It remains to prove Claims $\mathrm{A}$ and $\mathrm{B}$ for a proper, positive-dimensional face $F=F_{S \backslash U}(x)$ of $[0,1]^{S}$. If $F$ is an opposite face, then Claim A holds by hypothesis and Claim B is vacuous.

Now assume that $F$ is not an opposite face. Assume that $X$ is persistent relative to the boundary $\partial F$ of the face. Let $f: I \rightarrow(0,1)^{S}$ be a trajectory of $X$, and let $d_{1}=\mathrm{d}(f(\inf I), F)$ be the distance to $F$ from the initial point of the trajectory. The goal is to exhibit $\varepsilon>0$ so that the trajectory remains at distance greater than $\varepsilon$ from $F$; that is, $\mathrm{d}(f(I), F) \geq \varepsilon$. Claim A then follows as a consequence.

By hypothesis, $X$ is persistent relative to the boundary $\partial F$, so there exists $d_{2}>0$ such that $\mathrm{d}(f(I), \partial F) \geq d_{2}$. Decreasing $d_{2}$ if necessary, assume that $d_{2} \leq d_{1}$ and that $d_{2} / 2 \leq \varepsilon^{\prime}$, where $\varepsilon^{\prime}>0$ is such that trajectories in the block $F_{\varepsilon^{\prime}}$ can be projected (Definition 3.13).

Consider the block $F_{d_{2} / 2}$ of $F$. If the image of the trajectory $f(I)$ fails to intersect the block $F_{d_{2} / 2}$, then by defining $\varepsilon=d_{2} / 2$ it follows that $\mathrm{d}(f(I), F)>\varepsilon$, and we are done. Therefore, we can and do assume that $I^{\prime} \subseteq I$ is a maximal nonempty subinterval such that $f\left(I^{\prime}\right) \subseteq F_{d_{2} / 2}$, and let $\iota=f\left(\inf I^{\prime}\right)$ denote the corresponding initial point, as in the following illustration.

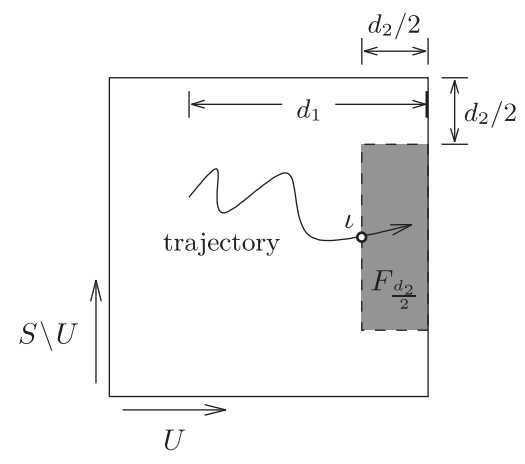

Note that $\mathrm{d}(\iota, F)=d_{2} / 2$. Indeed, by maximality of the interval $I^{\prime}$ the point $\iota$ lies on the boundary of $F_{d_{2} / 2}$, and the only boundary face of $F_{d_{2} / 2}$ that intersects the interior $(0,1)^{S}$ of the hypercube without also being contained in the $d_{2}$-neighborhood of the boundary $\partial F$ has constant distance $d_{2} / 2$ from $F$; see the following illustration.

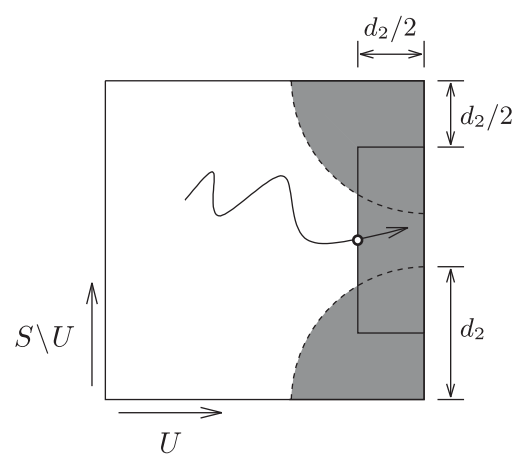

Because $\mathcal{X}$ is a vertexical family, there exist a differential inclusion $Y$ in $\mathcal{X}_{U}$, a trajectory $g: J \rightarrow[0,1]^{U}$ of $Y$, and an order-preserving continuous map $\alpha: I^{\prime} \rightarrow J$ such that $g \circ \alpha=$ 
$\pi_{U} \circ f$ with domain $I^{\prime}$. By definition, $Y$ depends only on $X$ and $F$, and not on the particular trajectory $f$ or the subinterval $I^{\prime}$. To prove Claim A for this face $F$, it now suffices to show that there exists $\varepsilon>0$, depending only on $d_{2}$, such that $\mathrm{d}\left(g(J), \pi_{U}(F)\right) \geq \varepsilon$, for this claim implies that $\mathrm{d}(f(I), F) \geq \varepsilon$, as desired.

Since $F$ is not an opposite face, by definition it contains a charged vertex, which we assume without loss of generality is $x$ (recall that $F=F_{S \backslash U}(x)$ ). We claim that $y=\pi_{U}(F)$ is a charged vertex of $[0,1]^{U}$; that is, $y_{i}=0$ for all $i \in U \backslash R$. Indeed, $j \in U$ implies $x_{j}=y_{j}$ because $x, y \in F=F_{S \backslash U}(x)$, and if additionally $j \notin R$, then $x_{j}=0$ because $x$ is charged.

By hypothesis of the theorem, $Y$ is repelled by the charged vertex $y=\pi_{U}(F)$ of $[0,1]^{U}$. Hence there exists $\varepsilon>0$ such that all trajectories of $Y$ starting at distance $d_{2} / 2$ away from the vertex $\pi_{U}(F)$ - and in particular, the trajectory $g$, because $\iota$ has distance precisely $d_{2} / 2$ from $F$ - never get closer than $\varepsilon$ to the vertex $\pi_{U}(F)$. This choice of $\varepsilon$ depends only on the distance $d_{2} / 2$, not on any aspect of the particular trajectory $f$; its existence proves Claim A.

To prove Claim B for this face $F$, assume $X$ is repelled by $\partial F$, and let $O_{1} \subseteq[0,1]^{S}$ be an open set containing $F$. Using that $X$ is also persistent relative to $\partial F$, repeat the argument above, but with $d_{1}>0$ now denoting the distance from $[0,1]^{S} \backslash O_{1}$ to $F$. The assumption that $X$ is repelled by $\partial F$ implies that the value of $d_{2}$ as found above depends only on $d_{1}$ and not on any aspect of any trajectory $f$ that begins outside $O_{1}$. Thus, the value of $\varepsilon$ (as above) depends only on $d_{2}$, which in turn depends only on $d_{1}$. Trajectories of $X$ starting outside $O_{1}$ therefore remain at distance at least $\varepsilon$ from $F$. Hence $X$ is repelled by $F$, as per Remark 2.11, proving Claim B.

\subsection{Consequences, special cases, and clarifications}

Next, we give three corollaries of Theorem 3.15. First, when the repulsing index set $R$ consists of all positive integers $\mathbb{Z}_{\geq 1}$, the theorem specializes to the following statement.

Corollary 3.17. Fix a vertexical family $\mathcal{X}=\left\{\mathcal{X}_{S}\right\}_{S \in \mathcal{S}}$ on open hypercubes indexed by the set $\mathcal{S}$ of all finite nonempty subsets of the positive integers $\mathbb{Z}_{\geq 1}$. If for every set $S \in \mathcal{S}$, every differential inclusion $X \in \mathcal{X}_{S}$ is repelled by the vertex set $\{0,1\}^{S}$ of its hypercube, then every such $X$ is repelled by, and hence persistent relative to, the boundary $\partial[0,1]^{S}$.

In words, Corollary 3.17 states that to prove that every differential inclusion in a vertexical family is repelled by the boundary, it suffices to show that each such differential inclusion is repelled by the vertices. The intuition behind this result is as follows. If a trajectory remains near a proper face and away from its boundary for some time, then the vertexical property allows us to project that part of the trajectory to a trajectory of a lower-dimensional differential inclusion in which the projected face is a vertex, which is repelling by assumption; hence the original trajectory stays away from the original face.

The next corollary is applied in our subsequent work [15] to prove persistence results for differential inclusion families that arise from strongly endotactic reaction networks. When the repulsing index set $R$ is empty, Theorem 3.15.1 specializes to the following statement.

Corollary 3.18. Fix a vertexical family $\mathcal{X}=\left\{\mathcal{X}_{S}\right\}_{S \in \mathcal{S}}$ on open hypercubes indexed by the set $\mathcal{S}$ of all finite nonempty subsets of the positive integers $\mathbb{Z}_{\geq 1}$. Suppose that for every set $S \in \mathcal{S}$, every differential inclusion $X \in \mathcal{X}_{S}$ satisfies the following hypotheses:

1) $X$ is repelled by the origin of its hypercube $(0,1)^{S}$, and

2) $X$ is persistent relative to the union of all faces that do not contain the origin.

Then every $X \in \mathcal{X}_{S}$ is persistent relative to the boundary $\partial[0,1]^{S}$. 
Our final corollary is used to prove permanence-like results in our next work [15]. More precisely, Corollary 3.21 gives conditions under which trajectories of a differential inclusion $X$ that begin in a compact set $K$ never leave a larger compact set. For ease of notation, we now introduce the following differential inclusion.

Definition 3.19. In the setting of Definition 2.4, fix a differential inclusion $X \subseteq T M$ and a subset $K \subseteq M$. The restricted differential inclusion $X_{K} \subseteq X$ is the smallest differential inclusion such that every trajectory of $X$ that begins in $K$ is a trajectory of $X_{K}$.

In other words, $X_{K}$ consists of all tangent vectors to all trajectories of $X$ that begin in $K$.

Lemma 3.20. Fix a finite set $S$ and a compact set $K \subseteq(0,1)^{S}$. If a differential inclusion $X \subseteq T(0,1)^{S}$ is repelled by the boundary $\partial[0,1]^{S}$, then there exists a compact set $K^{+}$with $K \subseteq K^{+} \subseteq(0,1)^{S}$ such that no trajectory of $X$ that begins in $K$ leaves $K^{+}$.

Proof. The open set $O_{1}=[0,1]^{S} \backslash K$ contains the boundary $\partial[0,1]^{S}$, so by definition of repelling, there exists an open set $O_{2}$ in $[0,1]^{S}$ that also contains the boundary $\partial[0,1]^{S}$ such that trajectories of $X_{K}$ that begin in $K$ never leave the compact set $K^{+}=[0,1]^{S} \backslash O_{2}$. Hence, no trajectory of $X$ that begins in $K$ leaves $K^{+}$.

Corollary 3.21. Fix a vertexical family $\mathcal{X}=\left\{\mathcal{X}_{S}\right\}_{S \in \mathcal{S}}$ on open hypercubes indexed by the set $\mathcal{S}$ of all finite nonempty subsets of the positive integers $\mathbb{Z}_{\geq 1}$, and a repulsing index set $R \subseteq \mathbb{Z}_{\geq 1}$. Assume the hypotheses of Theorem 3.15. Fix a set $S \subseteq \mathcal{S}$, a compact set $K \subseteq(0,1)^{S}$, and a differential inclusion $X \in \mathcal{X}_{S}$. If $X_{K}$ is repelled by the union of all opposite faces of $[0,1]^{S}$, then there exists a compact set $K^{+}$with $K \subseteq K^{+} \subseteq(0,1)^{S}$ such that no trajectory of $X$ that begins in $K$ leaves $K^{+}$.

Proof. Immediate from Theorem 3.15.2 and Lemma 3.20.

If there exists $d>0$ so that all trajectories of $X$ starting in $K$ remain at distance greater than $d$ from all opposite faces, then $X_{K}$ is repelled by the union of the opposite faces. Consequently, in the context of Corollary 3.21, it follows that there exists a compact set $K^{+}$such that no trajectory of $X$ starting in $K$ leaves $K^{+}$. In particular, if the repulsing index set is the empty set, then the existence of such a bound $d$ simply means an upper bound $1-d \in(0,1)$ on all coordinate components of all trajectories of $X$ beginning in $K$.

Remark 3.22. The significance of Corollary 3.21 is that, given the flow from $K$, promoting its persistence relative to the opposite faces to repulsion by the opposite faces results in its repulsion by the entire boundary. In this form, it looks like a weaker form of Corollary 3.17, but restricted to those trajectories that begin in $K$. Note that it is a weaker form because in Corollary 3.21, we assume not only that $X_{K}$ is repelled by all vertices, but also that it is repelled by the union of all the opposite faces. An assumption like this appears to be necessary: without this assumption, the projections of opposite faces are vertices in lower-dimensional differential inclusions that need not be repelling (only for $X_{K}$ are the opposite faces assumed to be repelling).

Remark 3.23. In the statement of Theorem 3.15, if the goal is to prove that a particular differential inclusion $X \in \mathcal{X}_{S}$ is persistent relative to $\partial[0,1]^{S}$ (or repelled by the charged set), then it is enough to assume a slightly weaker hypothesis, namely that $(i) X$ itself is persistent relative to the union of all opposite faces and repelled by the charged vertices of the hypercube, and $(i i)$ the lower-dimensional set $\mathcal{X}_{U}$ for each proper nonempty subset $U \subseteq S$ is persistent relative to the union of all opposite faces and repelled by the charged vertices of its corresponding hypercube $[0,1]^{U}$. 
Remark 3.24. We devised the notion of "repelled by" (Definition 2.4) expressly for the purpose of proving Theorem 3.15. It is natural to ask whether this notion is necessary: is a vertexical family that is persistent relative to the charged vertices necessarily persistent relative to the boundary? In other words, in the statement of Theorem 3.15, can instances of "repelled by" be replaced by "persistent relative to"? The answer is no: such a replacement makes the theorem false. Using the hypothesis that certain vertices are repelling, in the proof of Theorem 3.15 one obtains a value $\varepsilon>0$ (for a neighborhood of the face) that depends only on $d_{2} / 2$ (the thickness of the block of the face). However, under the weaker assumption of persistence relative to the vertices, this value $\varepsilon$ depends on the specific subinterval $I^{\prime}$. A trajectory can repeatedly enter and exit the block, so that there are infinitely many relevant subintervals $I^{\prime}$. In this case, it is possible for the trajectory to enter arbitrarily small neighborhoods without violating persistence. Also recall from Remark 2.6 that although repulsion implies persistence, the converse is false.

\section{Reaction network theory}

In this section, we define reaction networks, reaction systems, and their properties.

\subsection{Reaction networks}

Our networks are more general than usual for chemical reaction network theory [13, 17].

Definition 4.1. Write OpnInt $=\{(a, b) \mid 0 \leq a<b \leq \infty\}$ for the set of open subintervals of $\mathbb{R}_{>0}$ and CmpctInt $=\{[a, b] \mid 0<a \leq b<\infty\}$ for the set of compact subintervals.

1. A reaction network $(S, \mathcal{C}, \mathcal{R})$ is a triple of finite sets: a set $S$ of species, a set $\mathcal{C} \subseteq \mathbb{R}^{S}$ of complexes, and a set $\mathcal{R} \subseteq \mathcal{C} \times \mathcal{C}$ of reactions.

2 . The reaction graph is the directed graph $(\mathcal{C}, \mathcal{R})$ whose vertices are the complexes and whose directed edges are the reactions.

3. A reaction $r=\left(y, y^{\prime}\right) \in \mathcal{R}$, also written $y \rightarrow y^{\prime}$, has reactant $y=\operatorname{reactant}(r) \in \mathbb{R}^{S}$, product $y^{\prime}=\operatorname{product}(r) \in \mathbb{R}^{S}$, and reaction vector

$$
\operatorname{flux}(r)=\operatorname{product}(r)-\operatorname{reactant}(r) .
$$

4. The reaction diagram is the realization $(\mathcal{C}, \mathcal{R}) \rightarrow \mathbb{R}^{S}$ of the reaction graph that takes each reaction $r \in \mathcal{R}$ to the translate of $\operatorname{flux}(r)$ that joins reactant $(r)$ to $\operatorname{product}(r)$.

5. A linkage class is a connected component of the reaction graph.

Remark 4.2. The chemical reaction network theory literature usually imposes the following requirements for a reaction network.

- Each complex takes part in some reaction: for all $y \in \mathcal{C}$ there exists $y^{\prime} \in \mathcal{C}$ such that $\left(y, y^{\prime}\right) \in \mathcal{R}$ or $\left(y^{\prime}, y\right) \in \mathcal{R}$.

- No reaction is trivial: $(y, y) \notin \mathcal{R}$ for all $y \in \mathcal{C}$.

Definition 4.1 does not impose these conditions; in other words, our reaction graphs may include isolated vertices or self-loops. We drop these conditions to ensure that the projection of a network - obtained by removing certain species - remains a network under our definition even if some reactions become trivial (see Definition 5.1.1). In addition, like Craciun, Nazarov, and Pantea $[10, \S 7]$, we allow arbitrary real complexes $y \in \mathbb{R}^{S}$, so our setting is more general than that of usual chemical reaction networks, whose complexes $y \in \mathbb{Z}_{\geq 0}^{S}$ are nonnegative integer combinations of species, as in the following definition. The ODE systems defined in the next subsection that result from real complexes have been studied over the years and called "power-law systems" (see Remark 5.17). 
Definition 4.3. A reaction network $(S, \mathcal{C}, \mathcal{R})$ is

1) integer if $\mathcal{C} \subseteq \mathbb{Z}^{S}$;

2) chemical if $\mathcal{C} \subseteq \mathbb{Z}_{\geq 0}^{S}$;

3) reversible if the reaction graph of the network is undirected: a reaction $\left(y, y^{\prime}\right)$ lies in $\mathcal{R}$ if and only if its reverse reaction $\left(y^{\prime}, y\right)$ also lies in $\mathcal{R}$;

4) strongly connected if the reaction graph of the network is strongly connected; that is, if the reaction graph contains a directed path between each pair of complexes;

5) weakly reversible if every linkage class of the network is strongly connected.

Note that a network is strongly connected if and only if it is weakly reversible and has only one linkage class.

The next definitions introduce endotactic networks of [10].

Definition 4.4. The standard basis of $\mathbb{R}^{S}$ indexed by $S$ defines a canonical inner product $\langle\cdot, \cdot\rangle$ on $\mathbb{R}^{S}$ with respect to which the standard basis is orthonormal. Let $w \in \mathbb{R}^{S}$.

1. The vector $w$ defines a preorder on $\mathbb{R}^{S}$, denoted by $\leq_{w}$, in which

$$
y \leq_{w} y^{\prime} \Leftrightarrow\langle w, y\rangle \leq\left\langle w, y^{\prime}\right\rangle
$$

Write $y<_{w} y^{\prime}$ if $\langle w, y\rangle<\left\langle w, y^{\prime}\right\rangle$.

2. For a finite subset $Y \subseteq \mathbb{R}^{S}$, denote by $\operatorname{init}_{w}(Y)$ the set of $\leq{ }_{w}$-maximal elements of $Y$ :

$$
\operatorname{init}_{w}(Y)=\left\{y \in Y \mid\langle w, y\rangle \geq\left\langle w, y^{\prime}\right\rangle \text { for all } y^{\prime} \in Y\right\} \text {. }
$$

3. For a reaction network $(S, \mathcal{C}, \mathcal{R})$, the set $\mathcal{R}_{w} \subseteq \mathcal{R}$ of $w$-essential reactions consists of those whose reaction vectors are not orthogonal to $w$ :

$$
\mathcal{R}_{w}=\{r \in \mathcal{R} \mid\langle w, \text { flux }(r)\rangle \neq 0\} .
$$

4. The $w$-support $\operatorname{supp}_{w}(S, \mathcal{C}, \mathcal{R})$ of the network is the set of vectors that are $\leq{ }_{w}$-maximal among reactants of $w$-essential reactions:

$$
\operatorname{supp}_{w}(S, \mathcal{C}, \mathcal{R})=\operatorname{init}_{w}\left(\operatorname{reactant}\left(\mathcal{R}_{w}\right)\right) .
$$

Remark 4.5. In order to simplify the computations in our next work [15], we differ from the usual convention $[10,20]$, by letting $\operatorname{init}_{w}(Y)$ denote the $\leq_{w}$-maximal elements rather than the $\leq_{w}$-minimal elements. Accordingly, the inequalities in Definition 4.6 are switched, so our definition of endotactic is equivalent to the usual one.

Definition 4.6. Fix a reaction network $(S, \mathcal{C}, \mathcal{R})$.

1. The network $(S, \mathcal{C}, \mathcal{R})$ is $w$-endotactic for some $w \in \mathbb{R}^{S}$ if

$$
\langle w, \operatorname{flux}(r)\rangle<0
$$

for all $w$-essential reactions $r \in \mathcal{R}_{w}$ such that reactant $(r) \in \operatorname{supp}_{w}(S, \mathcal{C}, \mathcal{R})$.

2. The network $(S, \mathcal{C}, \mathcal{R})$ is $W$-endotactic for a subset $W \subseteq \mathbb{R}^{S}$ if $(S, \mathcal{C}, \mathcal{R})$ is $w$-endotactic for all vectors $w \in W$.

3. The network $(S, \mathcal{C}, \mathcal{R})$ is endotactic if it is $\mathbb{R}^{S}$-endotactic. 
4. $(S, \mathcal{C}, \mathcal{R})$ is strongly endotactic if it is endotactic and for every vector $w$ that is not orthogonal to the stoichiometric subspace of $(S, \mathcal{C}, \mathcal{R})$, there exists a reaction $y \rightarrow y^{\prime}$ in $\mathcal{R}$ such that

(i) $y>_{w} y^{\prime}$, and

(ii) $y$ is $\leq_{w}$-maximal among all reactants in $(S, \mathcal{C}, \mathcal{R}): y \in \operatorname{init}_{w}(\operatorname{reactant}(\mathcal{R}))$.

Remark 4.7. Endotactic chemical reaction networks, which generalize weakly reversible networks, were introduced by Craciun, Nazarov, and Pantea $[10, \S 4]$. Our definition is slightly more general still, because we do not require the reaction networks to be chemical (Definition 4.6). Strongly endotactic reaction networks are new; they give rise to strong results concerning persistence using our techniques; see Theorem 6.11. Strongly connected networks (i.e., weakly reversible networks with only one linkage class) are strongly endotactic.

Remark 4.8. For the geometric intuition behind Definition 4.6, imagine a hyperplane normal to $w$ that is sweeping across the reaction diagram in $\mathbb{R}^{S}$ from "infinity in direction $w$ ". As this hyperplane sweeps, it stops when it first reaches the reactant $y$ of a reaction $y \rightarrow y^{\prime}$ that is not perpendicular to $w$. If all such reactions do not point into the halfspace already swept by the hyperplane - that is, all such reactions have product $y^{\prime}$ outside of the open swept halfspace then the network is $w$-endotactic. Equivalently, the network is endotactic if no such reaction makes an acute angle with $w$. Illustrations can be found in [10].

As for strongly endotactic networks, the sweeping hyperplane now stops when it first touches the reactant of any reaction, whether or not it is perpendicular to $w$. Again we require that the products of all such reactions lie outside of the open swept halfspace, and in addition at least one of these reactions is not perpendicular to $w$. If this condition is satisfied for all vectors $w$ not orthogonal to the stoichiometric subspace, then the network is strongly endotactic. Both endotactic and strongly endotactic networks capture the idea that extreme reactions should not point outward.

Example 4.9. Here we follow the usual convention of depicting a network by its reaction graph or reaction diagram and writing a complex as, for example, $2 A+B$ rather than $y=(2,1)$. The Lotka-Volterra reaction network, consisting of the three reactions

$$
A \rightarrow 2 A, \quad A+B \rightarrow 2 B, \quad B \rightarrow 0,
$$

is not endotactic. Reversing all three reactions yields the network

$$
2 A \rightarrow A, \quad 2 B \rightarrow A+B, \quad 0 \rightarrow B,
$$

which is strongly endotactic, as can be verified from its reaction diagram:

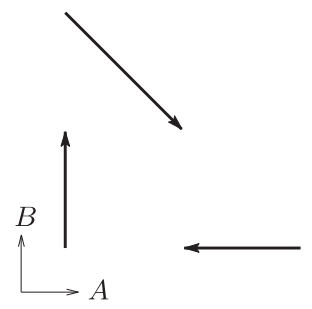

Example 4.10. Every weakly reversible reaction network is endotactic [10]. However, even a reversible reaction network may fail to be strongly endotactic, as in the following example of 
a pair of reversible reactions. For $w=(0,-1)$, the $\leq_{w}$-maximal reactant complexes are at the bottom, but both of the corresponding reactions are perpendicular to $w$.

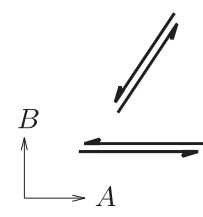

\subsection{Reaction systems}

Definition 4.11. The stoichiometric subspace $H$ of a network is the span of its reaction vectors. For a positive vector $x_{0} \in \mathbb{R}_{>0}^{S}$, the invariant polyhedron of $x_{0}$ is the polyhedron

$$
\mathcal{P}=\left(x_{0}+H\right) \cap \mathbb{R}_{\geq 0}^{S} .
$$

This polyhedron is also referred to as the stoichiometric compatibility class in the chemical reaction network theory literature [13].

Definition 4.12. Let $(S, \mathcal{C}, \mathcal{R})$ be a reaction network.

1. A tempering is a map $\kappa: \mathcal{R} \rightarrow$ CmpctInt that assigns to each reaction a nonempty compact positive interval.

2. A set $D \subseteq \mathbb{R}_{>0}^{S}$ is a domain if its intersection with every invariant polyhedron $\mathcal{P}$ of $(S, \mathcal{C}, \mathcal{R})$ is open in $\mathcal{P}$.

A reaction system is a triple consisting of a reaction network, a tempering, and a domain.

Remark 4.13. Mass-action differential inclusions of reaction systems (Definition 5.16) generalize the usual mass-action kinetics ODE systems; see Remark 5.17. One thinks of a domain as a promise that concentrations of species remain within the domain. To explain the motivation behind temperings, recall that a reaction network gives rise to a dynamical system by way of reaction rates. For biochemical reaction networks, one is typically unable to measure precise values for the rates. This occurs both because of incomplete information, and because of molecular and environmental variability. One way to model this uncertainty is to allow reaction rates $\kappa(r)$ to be time-dependent, as long as they are uniformly bounded away from 0 and $\infty$. Craciun, Nazarov, and Pantea called such systems $\kappa$-variable [10]. In a similar spirit, we have chosen to work with differential inclusions, allowing $\kappa(r)$ to take on every value from an appropriate interval.

\section{Definition 4.14.}

1. A confined reaction system is a reaction system whose domain is an invariant polyhedron of the underlying reaction network.

2. An allotment is a map $\mu: S \rightarrow$ OpnInt sending each species $s \in S$ to an open positive interval. The allotment hypercube of an allotment $\mu$ is the open hypercube $\square^{\mu}=\prod_{s \in S} \mu(s) \subseteq$ $\mathbb{R}_{>0}^{S}$. A subconfined reaction system is specified by a reaction system and an allotment, in which the domain of the reaction system is the intersection of the allotment hypercube and an invariant polyhedron of the underlying reaction network.

Remark 4.15. Every confined system is viewed as a subconfined system in which the allotment is understood to send every species $s$ to $(0, \infty)$, so the allotment hypercube is the entire positive orthant. 
Remark 4.16. The mathematical motivation prompting temperings and allotments is to ensure that projections of trajectories "stay in the family". Projections forget the exact concentrations of eliminated species. Absorbing the effect of pre-projection dynamics into post-projection dynamics requires a guarantee that the projected species concentrations never leave a certain sufficiently large interval. Therefore, in post-projection dynamics, the reaction rates remain within appropriately enlarged intervals. Allotments and tempering reaction rates provide the extra flexibility for this construction.

This intuition is made precise in Section 5: interval-valued rates allow us to define an appropriate domain category on which mass-action kinetics becomes functorial (Theorem 5.20). As a consequence, projective classes of reaction systems (Definition 5.1) give rise to families of differential inclusions that are vertexical.

Remark 4.17. Invariant polyhedra are forward-invariant sets with respect to the dynamics arising from mass-action kinetics; see Remark 5.19. Therefore, a confined reaction system allows us to restrict our attention to the dynamics on a specific invariant set.

\section{$5 \quad$ Functoriality of mass-action kinetics}

To every reaction system $N$ we assign a differential inclusion $\mathfrak{M}(N)$ (Definition 5.16). This assignment $\mathfrak{M}$ generalizes the usual mass-action kinetics ODE system in the chemical reaction network theory literature $[13,17]$. It is the goal of this section to analyze how $\mathfrak{M}$ behaves under projections of subconfined reaction systems. The main result (Theorem 5.23) states that projective classes of reaction systems (Definition 5.1) give rise to vertexical families of differential inclusions. In particular, chemical, reversible, weakly reversible, endotactic, and strongly endotactic reaction systems all give rise to vertexical families (Corollary 5.24).

The first task, which occupies Section 5.1, is to make precise what is meant by projection, and by maps between differential inclusions. It then becomes routine to verify two properties that are key to the proof of Theorem 5.23, namely that for every pair of subconfined reaction systems $N_{1}$ and $N_{2}$ such that $N_{2}=p\left(N_{1}\right)$ is a projection of $N_{1}$, the assignment $\mathfrak{M}$ induces a map $\mathfrak{M}(p): \mathfrak{M}\left(N_{1}\right) \rightarrow \mathfrak{M}\left(N_{1}\right)$ between the corresponding differential inclusions such that

1) the identity projection gets sent to the identity map on differential inclusions, and

2) the composition $p=p_{2} \circ p_{1}$ of two projection maps $p_{1}: N_{1} \rightarrow N_{2}$ and $p_{2}: N_{2} \rightarrow N_{3}$ gets sent to the composition $\mathfrak{M}\left(p_{2}\right) \circ \mathfrak{M}\left(p_{1}\right)$ of the corresponding maps between differential inclusions; that is,

$$
\mathfrak{M}(p)=\mathfrak{M}\left(p_{2}\right) \circ \mathfrak{M}\left(p_{1}\right)
$$

These two properties of $\mathfrak{M}$ are precisely the ones required by the definition of a functor in category theory. Therefore, we find it economical to use this language (Theorem 5.20). Readers unfamiliar with the language of category theory should read the word "functor" as shorthand for the two properties. This is the extent of the category theory used in this paper.

\subsection{Categorical definitions}

Definition 5.1. Recall, from Definition 3.10, the projection $\pi_{U}: \mathbb{R}^{S} \rightarrow \mathbb{R}^{U}$ for $U \subseteq S$, and denote by $\pi_{U}^{\times 2}=\pi_{U} \times \pi_{U}: \mathbb{R}^{S} \times \mathbb{R}^{S} \rightarrow \mathbb{R}^{U} \times \mathbb{R}^{U}$ the product of $\pi_{U}$ with itself.

1. For a reaction network $(S, \mathcal{C}, \mathcal{R})$, and a nonempty subset $U \subseteq S$ of species, the reduced reaction network is the reaction network $\pi_{U}(S, \mathcal{C}, \mathcal{R})=\left(U, \pi_{U}(\mathcal{C}), \pi_{U}^{\times 2}(\mathcal{R})\right)$. 
2. A property $P$ of reaction networks is projective if for all finite nonempty sets $S$, reaction networks $(S, \mathcal{C}, \mathcal{R})$, and nonempty subsets $U \subseteq S$, if $(S, \mathcal{C}, \mathcal{R})$ has property $P$ then the reduced reaction network $\pi_{U}(S, \mathcal{C}, \mathcal{R})$ has property $P$.

3. The set of all reaction networks with a given projective property is a projective class.

Remark 5.2. The reduced reaction network $\pi_{U}(S, \mathcal{C}, \mathcal{R})$ is obtained from the reaction network $(S, \mathcal{C}, \mathcal{R})$ by deleting all species outside of $U$. This concept was defined by Anderson [2, $\S 3.2]$, who required that any trivial reactions be removed from the reduced reaction set $\left(\pi_{U} \times \pi_{U}\right)(\mathcal{R})$. In contrast, we allow trivial reactions (Remark 4.2). Another related notion in the context of reversible reactions is that of "reduced event-system" introduced in [1].

Example 5.3. For $U=\{A\}$, the reduced network

$$
2 A \rightarrow A \leftarrow 0 \leftarrow 0
$$

of the network (1) in Example 4.9 is obtained by removing species $B$. The reduced network is strongly endotactic, as is the original network (1).

Next we see that the implication in Example 5.3 holds in general for strongly endotactic networks. Such an implication was already completed for weakly reversible networks by Anderson [2, Lemma 3.4], and for endotactic networks by Pantea [20, Proposition 3.1].

Lemma 5.4. The classes of integer, chemical, reversible, strongly connected, weakly reversible, endotactic, or strongly endotactic reaction networks are projective. Further, if $P_{1}$ and $P_{2}$ are projective properties, then so are the conjunction $P_{1} \wedge P_{2}$ and disjunction $P_{1} \vee P_{2}$.

Proof. Projectivity holds for integer and chemical networks because projection preserves integrality and nonnegativity of points in $\mathbb{R}^{S}$. Projectivity holds for reversible, strongly connected, and weakly reversible networks because these conditions depend only on the reaction graph, on whose vertices and edges projection is surjective.

Next, consider an endotactic network with species set $S$ and the reduced network arising from a nonempty subset $U \subseteq S$. Take any vector $w \in \mathbb{R}^{U}$. For any reduced reaction $\pi_{U}(r)$, where $r$ is a reaction in the original network,

$$
\left\langle w, \operatorname{flux}\left(\pi_{U}(r)\right)\right\rangle=\langle(w, 0), \operatorname{flux}(r)\rangle .
$$

Thus, the $w$-essential reactions of the reduced network are the projections under $\pi_{U}^{\times 2}$ of the $(w, 0)$-essential reactions of the original network, where we write $(w, 0) \in \mathbb{R}^{U} \times \mathbb{R}^{S \backslash U}$. Similarly, the $w$-support of the reduced network is the projection under $\pi_{U}$ of the $(w, 0)$-support of the original network. So, if $\pi_{U}(r)$ is a $w$-essential reaction of the reduced network with reactant in the $w$-support, then the original reaction $r$ is a $(w, 0)$-essential reaction of the original network with reactant in the $(w, 0)$-support. By $(3)$ and the definition of endotactic, $\left\langle w\right.$, flux $\left.\left(\pi_{U}(r)\right)\right\rangle=$ $\langle(w, 0), \operatorname{flux}(r)\rangle<0$. Hence the reduced network is endotactic.

Next, let $H$ denote the stoichiometric subspace of a strongly endotactic network, so $\pi_{U}(H)$ is the stoichiometric subspace of the reduced network. Take a vector $w \in \mathbb{R}^{U}$ that is not orthogonal to $\pi_{U}(H)$. We need only show that there exists a reaction $\pi_{U}(y) \rightarrow \pi_{U}\left(y^{\prime}\right)$, where $y \rightarrow y^{\prime}$ is a reaction in the original network, such that $\pi_{U}(y)>_{w} \pi_{U}\left(y^{\prime}\right)$ and $\pi_{U}(y)$ is $\leq_{w}$-maximal among all reactant vectors in the reduced network. Again consider $(w, 0) \in \mathbb{R}^{U} \times \mathbb{R}^{S \backslash U}$. As $w$ is not orthogonal to $\pi_{U}(H)$, it follows that $(w, 0)$ is not orthogonal to $H$, and the preorder $\leq_{w}$ on the reduced reactant complexes is the projection under $\pi_{U}$ of the preorder $\leq_{(w, 0)}$ on the original reactant complexes. Since the original network is strongly endotactic, there is a reaction $y \rightarrow y^{\prime}$ in the original network with $y>_{(w, 0)} y^{\prime}$ such that $y$ is $\leq_{(w, 0)}$-maximal among all reactant vectors. This reaction achieves our requirements.

The claim about conjunctions and disjunctions follows formally by Definition 5.1.2. 
Notation 5.5. Let $I, J \subseteq \mathbb{R}_{\geq 0}$ be two intervals, and $S$ a finite nonempty set.

1. Define $I \cdot J=\{i \cdot j \mid i \in I, j \in J\} \subseteq \mathbb{R}_{>0}$ and $\bigodot_{s \in S} I_{s}$ pointwise.

2. For $n \in \mathbb{Z}_{\geq 1}$ define $I^{n}$ recursively as $I \cdot I^{n-1}$ with $I^{1}=I$.

3. $I \times J$ and $\prod_{s \in S} I_{s}$ denote Cartesian products of intervals, as usual.

Definition 5.6. Let $S$ be a finite nonempty set, and consider a function $\mu: S \rightarrow$ OpnInt to the set of open positive intervals. A nonempty subset $U \subseteq S$ is $\mu$-projectable if $0<\inf \mu(s)$ and $\sup \mu(s)<\infty$ for all $s \in S \backslash U$; that is, the left and right endpoints of the intervals $\mu(s)$ are bounded away from 0 and $\infty$ for those $s$ outside of $U$.

Remark 5.7. The condition of Definition 5.6 is on the complement $S \backslash U$ because those are the species removed in projecting to $U$, and so it is those species that must be bounded away from 0 and $\infty$. The set $S$ itself is trivially $\mu$-projectable, for all $\mu: S \rightarrow$ OpnInt.

We show that subconfined reaction systems form a category whose morphisms are projections, where the projection $p_{U}$ from one object $N$ to another corresponds to substituting intervals from the allotment of $N$ in place of a set $S \backslash U$ of forgotten species.

Definition 5.8. The category $\mathcal{N}$ of subconfined reaction systems with projections is given by the following data.

1. Objects: each is a subconfined reaction system $N$, specified by a reaction network $(S, \mathcal{C}, \mathcal{R})$ along with a tempering $\kappa: \mathcal{R} \rightarrow$ CmpctInt, an allotment $\mu: S \rightarrow$ OpnInt, and an invariant polyhedron $\mathcal{P}=\left(x_{0}+H\right) \cap \mathbb{R}_{\geq 0}^{S}$.

2. Morphisms: $p_{U}: N \rightarrow N^{\prime}$ if

- the network of $N^{\prime}$ is $\left(S^{\prime}, \mathcal{C}^{\prime}, \mathcal{R}^{\prime}\right)=\pi_{U}(S, \mathcal{C}, \mathcal{R})$ for a $\mu$-projectable subset $U \subseteq S$;

- the tempering of $N^{\prime}$ is $\kappa^{\prime}: \pi_{U}(r) \mapsto \kappa(r) \cdot \bigodot_{s \in S \backslash U} \mu(s)^{\text {reactant }(r)_{s}}$, where the exponent on $\mu(s)$ is the component indexed by $s$ in the vector reactant $(r)$;

- the allotment of $N^{\prime}$ is $\mu^{\prime}=\left.\mu\right|_{U}$, gotten by restricting the allotment of $N$ to $U$; and

- the invariant polyhedron of $N^{\prime}$ is $\mathcal{P}^{\prime}=\left(\pi_{U}\left(x_{0}\right)+\pi_{U}(H)\right) \cap \mathbb{R}_{\geq 0}^{U}$.

Remark 5.9. In Definition 5.8.2, $\pi_{U}(H)$ is the stoichiometric subspace of $N^{\prime}$ because it is spanned by the vectors flux $\left(\pi_{U}(r)\right)=\pi_{U}(\operatorname{flux}(r))$, where $r$ is a reaction of $N$. Thus, $\left(\pi_{U}\left(x_{0}\right)+\pi_{U}(H)\right) \cap \mathbb{R}_{\geq 0}^{U}$ is an invariant polyhedron of $N^{\prime}$.

Remark 5.10. Composition in $\mathcal{N}$ is well-defined because first projecting to $U \subseteq S$ and then projecting to $V \subseteq U$ is the same as projecting directly to $V$.

Mass-action kinetics assigns to each subconfined reaction system a differential inclusion on its domain. Theorem 5.20 states that this assignment makes mass-action kinetics a functor, with domain category $\mathcal{N}$ and codomain category as follows.

Definition 5.11. The category $\mathcal{D} \mathcal{I}$ of differential inclusions is given by the following data.

1. Objects: each is a choice of manifold with corners and a differential inclusion on it.

2. Morphisms: a morphism from $X \subseteq T M$ to $Y \subseteq T N$ is a continuous map $k: M \rightarrow N$ such that for each trajectory $f: I \rightarrow M$ of $X$, there is a trajectory $g: J \rightarrow N$ of $Y$ and an order-preserving continuous map $\alpha: I \rightarrow J$ satisfying

$$
k \circ f=g \circ \alpha .
$$


Lemma 5.12. Composition of continuous maps induces a well-defined composition on $\mathcal{D} \mathcal{I}$. Specifically, assume $X_{j} \subseteq T M_{j}$ for $j=1,2,3$ are differential inclusions, with morphisms $k_{12}$ : $M_{1} \rightarrow M_{2}$ and $k_{23}: M_{2} \rightarrow M_{3}$ in $\mathcal{D} \mathcal{I}$. If $f_{1}: I_{1} \rightarrow M_{1}$ is a trajectory of $X_{1}$, then there is a trajectory $f_{3}: I_{3} \rightarrow M_{3}$ of $X_{3}$ and an order-preserving continuous map $\alpha_{13}: I_{1} \rightarrow I_{3}$ such that the composite continuous map $k_{13}=k_{23} \circ k_{12}$ satisfies $k_{13} \circ f_{1}=f_{3} \circ \alpha_{13}$.

Proof. Given $f_{1}$, since $k_{12}$ is a morphism in $\mathcal{D I}$, there is a trajectory $f_{2}: I_{2} \rightarrow M_{2}$ of $X_{2}$ and a continuous order-preserving map $\alpha_{12}: I_{1} \rightarrow I_{2}$ such that $k_{12} \circ f_{1}=f_{2} \circ \alpha_{12}$. For the desired trajectory $f_{3}: I_{3} \rightarrow M_{3}$ of $X_{3}$ use the one afforded by virtue of $k_{23}$ being a morphism in $\mathcal{D I}$, given $f_{2}$, which comes with a continuous order-preserving map $\alpha_{23}: I_{2} \rightarrow I_{3}$ such that $k_{23} \circ f_{2}=f_{3} \circ \alpha_{23}$. Set $\alpha_{13}=\alpha_{23} \circ \alpha_{12}$. Then

$$
k_{13} \circ f_{1}=k_{23} \circ k_{12} \circ f_{1}=k_{23} \circ f_{2} \circ \alpha_{12}=f_{3} \circ \alpha_{23} \circ \alpha_{12}=f_{3} \circ \alpha_{13},
$$

as desired.

Remark 5.13. Compare the notion of morphism in $\mathcal{D} \mathcal{I}$ (Definition 5.11) with that of vertexical family (Definition 3.13). Equation (4) also occurs in Definition 3.13, with $k$ being a particular type of continuous map $\pi_{U}$. However, Definition 5.11 asks for a global map $k$, whereas the maps in Definition 3.13 are required only locally, on blocks of faces.

Remark 5.14. One motivation for defining the category of differential inclusions this way comes from the dynamical systems concept of topological equivalence [18], which identifies two phase portraits as qualitatively the same, even if the details of the dynamics may differ. The isomorphisms in our category $\mathcal{D} \mathcal{I}$ correspond exactly to topological equivalence.

For this reason, morphisms between differential inclusions may also be called topological morphisms. Intuitively, if a topological morphism is a monomorphism, then its target differential inclusion qualitatively simulates the domain differential inclusion. Maps that are not monomorphisms can of course result in the loss of information, in general. The categorical message of Theorem 3.15 is that it is sometimes possible to piece together many "lossy" maps on the same domain to regain substantial information about the domain dynamics.

Another concept from dynamical systems, topological conjugacy [18], is a stronger notion than topological equivalence that disallows time reparameterization. This motivates looking at a subcategory $\mathcal{D} \mathcal{I}_{1}$ of $\mathcal{D} \mathcal{I}$ in which the order-preserving map $\alpha$ in Definition 5.11 is required to be the identity map; the definition follows.

Definition 5.15. The category $\mathcal{D I}_{1}$ of differential inclusions with topological semiconjugacy morphisms is the subcategory of $\mathcal{D} \mathcal{I}$ with the following data.

1. Objects: the same objects as in $\mathcal{D} \mathcal{I}$.

2. Morphisms: a morphism from $X \subseteq T M$ to $Y \subseteq T N$ is a continuous map $k: M \rightarrow N$ such that $k \circ f$ is a trajectory of $Y$ whenever $f: I \rightarrow M$ is a trajectory of $X$.

The proof of Lemma 5.12 makes it plain that any composition in $\mathcal{D I}$ of morphisms in $\mathcal{D I}_{1}$ is a morphism in $\mathcal{D} \mathcal{I}_{1}$, because every reparameterization map $\alpha_{i j}$ in that proof can be taken to be the identity map on $I_{1}$.

The next definition uses the multinomial notation $x^{y}:=x_{1}^{y_{1}} x_{2}^{y_{2}} \cdots x_{m}^{y_{m}}$ for $x, y \in \mathbb{R}^{m}$.

Definition 5.16. The mass-action differential inclusion of a reaction system, specified by a reaction network $(S, \mathcal{C}, \mathcal{R})$ with tempering $\kappa$ and domain $D$, is the differential inclusion on $\mathbb{R}_{>0}^{S}$ whose fiber over each point $x \in D$ is

$$
\left\{\sum_{r \in \mathcal{R}} k_{r} x^{\text {reactant }(r)} \text { flux }(r) \mid k_{r} \in \kappa(r) \text { for all } r \in \mathcal{R}\right\} \subseteq \mathbb{R}^{S}=T_{x} \mathbb{R}_{>0}^{S}
$$


and whose fiber over all points $x \in \mathbb{R}_{>0}^{S} \backslash D$ is empty. The mass-action functor $\mathfrak{M}: \mathcal{N} \rightarrow \mathcal{D} \mathcal{I}_{1}$ from the category $\mathcal{N}$ of subconfined reaction systems to the category $\mathcal{D I}_{1}$ is defined on

1) objects $N \in \mathcal{N}$ by letting $\mathfrak{M}(N)$ be the mass-action differential inclusion of $N$, and on

2) morphisms $p_{U}: N \rightarrow N^{\prime}$ by letting $\mathfrak{M}\left(p_{U}\right)$ be the projection $\pi_{U}: \square^{\mu} \rightarrow \square^{\mu^{\prime}}$.

Remark 5.17. The usual mass-action kinetics ODE systems are the special cases in which the reaction network is chemical, the tempering $\kappa$ assigns not an interval of positive length but a point - the reaction rate constant - to each reaction, and the allotment hypercube is the entire positive orthant: $\square^{\mu}=\mathbb{R}_{>0}^{S}$. The more general setting where stoichiometric coefficients are allowed to be arbitrary (positive or negative) real numbers has been studied in biochemical systems theory under the names "power-law" and "generalized mass-action". This research area goes back to early work of Savageau in the 1960s [21].

Remark 5.18. In the next subsection, we use a diffeomorphism $\ell^{S}: \mathbb{R}_{>0}^{S} \rightarrow(0,1)^{S}$ to push forward mass-action differential inclusions to be defined on hypercubes rather than orthants (Definition 5.22). Therefore, for mass-action differential inclusions, the closure $\bar{M}$ of the allotment hypercube $M=\square^{\mu}$ is taken in the compactification $[0, \infty]^{S}$ when we are interested in the properties of being persistent, permanent, or repelled.

Remark 5.19. In a mass-action differential inclusion, only points in a specified domain have nonempty fiber. Imagine that we had instead defined a larger differential inclusion, so that all fibers in the positive orthant are nonempty and take the form given by mass-action (5). Then, recalling that the stoichiometric subspace of a network is spanned by the reaction vectors flux $(r)$, it follows from (5) that every trajectory of this larger differential inclusion would be confined to some invariant polyhedron (2). (This also uses the fact that trajectories remain nonnegative $[6, \S 2]$.) That is, the invariant polyhedra are forward-invariant with respect to the larger differential inclusion. Therefore it is in fact appropriate to restrict the differential inclusion to a given invariant polyhedron (as we did in Definition 5.16) and then to analyze properties such as persistence of the restriction.

\subsection{Functorial results and consequences}

Theorem 5.20. The mass-action functor $\mathfrak{M}$ is a functor from the category $\mathcal{N}$ of subconfined reaction systems with projection morphisms to the category $\mathcal{D} \mathcal{I}_{1}$ of differential inclusions with topological semiconjugacy morphisms.

Proof. The content of the statement is twofold: first, that $\mathfrak{M}\left(p_{U}\right)=\pi_{U}$ in Definition 5.16.2 indeed defines a morphism $\mathfrak{M}(N) \rightarrow \mathfrak{M}\left(N^{\prime}\right)$, and second, that $\mathfrak{M}$ preserves identity morphisms as well as compositions. The second is straightforward (projections are sent to projections). For the first, it suffices to observe that for any nonempty $U \subseteq S$, the projection $\pi_{U} \circ f$ of a trajectory $f$ of $\mathfrak{M}(N)$ is a trajectory of $\mathfrak{M}\left(p_{U}(N)\right)$ by definition of $p_{U}$. This observation uses the fact that the image of $f$ is in the invariant polyhedron $\mathcal{P}$ of $N$, so the image of $\pi_{U} \circ f$ is in $\pi_{U}(\mathcal{P})$ which is contained in the invariant polyhedron of $p_{U}(N)$.

Remark 5.21. When we fix an allotment, we do not, and can not, insist that trajectories stay within the allotment for all time. Our assertion of functoriality is to the effect that for the period of time that a trajectory does stay within the allotment, its projection factors through a smaller system. The definition of vertexical family (Definition 3.13) is weak enough to tolerate such a weak guarantee, and yet strong enough to be able to prove theorems on persistence and permanence. In fact, the definition of vertexical requires even less: the projection of a trajectory is required to factor through a smaller system only for the period of time that it lies in a corresponding block. 
Vertexical families are defined in terms of differential inclusions on unit hypercubes $(0,1)^{S}$. In contrast, the mass-action functor produces differential inclusions on positive orthants $\mathbb{R}_{>0}^{S}$. To translate back and forth, our next definition fixes a smooth, order-preserving diffeomorphism $\mathbb{R}_{>0} \rightarrow(0,1)$. The actual choice is irrelevant for our purposes, but if it helps, the reader may consider the function $x \mapsto x /(1+x)$.

Definition 5.22. Fix a smooth, order-preserving diffeomorphism $\ell: \mathbb{R}_{>0} \rightarrow(0,1)$. For every nonempty finite set $S$, let $\ell^{S}: \mathbb{R}_{>0}^{S} \rightarrow(0,1)^{S}$, with derivative $d \ell^{S}: T \mathbb{R}_{>0}^{S} \rightarrow T(0,1)^{S}$.

1. The pushforward of a differential inclusion $X$ on $\mathbb{R}_{>0}^{S}$ under $\ell$ is the differential inclusion $d \ell^{S}(X)$ on $(0,1)^{S}$.

2. For a subconfined reaction system $N$ defined on a species set $S$ with allotment $\mu$, the differential inclusion $\mathfrak{M}^{\ell}(N)$ is the pushforward of the mass-action differential inclusion $\mathfrak{M}(N)$, considered as a differential inclusion on the image $\ell^{S}\left(\square^{\mu}\right) \subseteq(0,1)^{S}$ of the allotment hypercube $\square^{\mu}$ under $\ell$.

The following is the main result of this section.

Theorem 5.23. Fix a projective property $P$ of reaction networks. The class $\mathcal{F}_{P}$ of all confined reaction systems whose underlying reaction networks have property $P$ yields a vertexical family $\mathfrak{M}^{\ell}\left(\mathcal{F}_{P}\right)=\left\{\mathfrak{M}^{\ell}(N) \mid N \in \mathcal{F}_{P}\right\}$ of differential inclusions on open hypercubes.

Proof. By definition, $\mathfrak{M}^{\ell}\left(\mathcal{F}_{P}\right)$ is a family of differential inclusions on open hypercubes. Hence we need only prove that $\mathfrak{M}^{\ell}\left(\mathcal{F}_{P}\right)$ is vertexical. Consider a confined reaction system $N \in \mathcal{F}_{P}$, specified by a reaction network $(S, \mathcal{C}, \mathcal{R})$ with tempering $\kappa$ and invariant polyhedron $\mathcal{P}$. Fix a proper nonempty subset $U \subseteq S$ and a vertex $x \in\{0,1\}^{S}$ of the hypercube $(0,1)^{S}$. Denote by $F=F_{S \backslash U}(x)$ the corresponding face of the hypercube.

Fix $0<\varepsilon<\frac{1}{2}$, and define $\mu^{\prime}=\mu_{\varepsilon, U}^{\prime}: S \rightarrow$ OpnInt by

$$
\mu^{\prime}(s)= \begin{cases}(0, \infty) & \text { if } s \in U, \\ \ell^{-1}(\varepsilon, 1-\varepsilon) & \text { if } s \in S \backslash U .\end{cases}
$$

Denote by $N^{\prime}$ the subconfined reaction system $N^{\prime}$ that agrees with $N$ except that the allotment of $N^{\prime}$ is $\mu^{\prime}$. The underlying reaction network of $N^{\prime}$ still has property $P$, because $N$ and $N^{\prime}$ have the same underlying reaction network.

While $N^{\prime}$ itself no longer has the entire positive orthant $\mathbb{R}_{>0}^{S}$ as its allotment hypercube, the set $U$ is $\mu^{\prime}$-projectable (Definition 5.6), and the reduced network $p_{U}\left(N^{\prime}\right)$ has allotment hypercube $\mathbb{R}_{>0}^{U}$. Since $P$ is projective, $p_{U}\left(N^{\prime}\right)$ therefore lies in $\mathcal{F}_{P}$. Consequently, by definition of vertexical family (Definition 3.13), it is enough to show that for any trajectory $f: I \rightarrow F_{\varepsilon}$ of $\mathfrak{M}^{\ell}(N)$ with image in the block $F_{\varepsilon}$, the projection $\pi_{U} \circ f=g$ is a trajectory of the mass-action differential inclusion $\mathfrak{M}^{\ell}\left(p_{U}\left(N^{\prime}\right)\right)$.

The trajectory $f$ is also a trajectory of $\mathfrak{M}^{\ell}\left(N^{\prime}\right)$, since $F_{\varepsilon} \subseteq \ell^{S}\left(\square^{\mu^{\prime}}\right)$ by construction of $\mu^{\prime}$. The result is now deduced easily from functoriality of mass-action kinetics (Theorem 5.20): the morphism $\pi_{U}$ in the category $\mathcal{D} \mathcal{I}_{1}$ from $\mathfrak{M}\left(N^{\prime}\right)$ to $\mathfrak{M}\left(p_{U}\left(N^{\prime}\right)\right)$ yields the pushforward morphism $\mathfrak{M}^{\ell}\left(N^{\prime}\right) \rightarrow \mathfrak{M}^{\ell}\left(p_{U}\left(N^{\prime}\right)\right)$ in $\mathcal{D} \mathcal{I}_{1}$, so the proof is complete by definition of morphisms in the category $\mathcal{D I}_{1}$ (Definition 5.15.2).

Recall that in propositional logic, a monotone (or monotonic) formula is one formed by the application of AND and OR operations only, without the use of NOT operations.

Corollary 5.24. Each of the classes of (monotone combinations of) integer, chemical, reversible, strongly connected, weakly reversible, endotactic, or strongly endotactic confined reaction systems generates a vertexical family of differential inclusions.

Proof. Immediate from Theorem 5.23 and Lemma 5.4. 
Remark 5.25. The category $\mathcal{N}$ of confined reaction systems with projections suffices for our purposes, but it would be more natural to allow arbitrary reaction systems with an associated domain set $D \subseteq \mathbb{R}_{>0}^{S}$ that is not necessarily derived from a Cartesian product of intervals. In addition, it is tempting to add to the category more morphisms, such as those corresponding to translation of the reaction diagram within $\mathbb{R}^{S}$, or scaling, rotation, arbitrary linear maps, graph homomorphisms, inversion $z=1 / x$, and so on. It is easy to verify that translation acts as a time-reparametrization. Hence, even allowing translations, mass-action kinetics remains a functor to $\mathcal{D} \mathcal{I}$. This leads to the following question, which we leave open.

Question 5.26. What is the richest domain category for which mass-action kinetics remains a functor to the category $\mathcal{D} \mathcal{I}$ of differential inclusions?

This question is important because a richer domain category would imply more ways of reducing the behavior of a network's mass-action kinetics to the behaviors of related networks. This could allow us to "program" (and analyze) instances of reaction dynamics in high dimensions as appropriate combinations of simpler reaction dynamics.

Remark 5.27. Consider a vertexical family of mass-action differential inclusions for which the one-dimensional differential inclusions in the family are known to be permanent. For instance, the differential inclusions arising from weakly reversible, endotactic, or strongly endotactic networks have this attribute. It is tempting to attempt to argue that such a family is permanent by the following induction: given a trajectory, all of its one-dimensional projections (which are also in the family, due to the functoriality of mass-action kinetics and projectivity of the relevant properties) are permanent, and hence the trajectory itself eventually remains in a compact set. However, this does not work because of uniformity issues. It is true that for a given trajectory, there exists a compact set that it enters eventually. However, what we need is one compact set so that every trajectory eventually enters this set, and our inductive argument does not prove this. A successful argument about permanence would require additional structure; for example, see Remark 6.7.

\section{Implications for persistence of mass-action systems}

One of the long-standing open problems of chemical reaction network theory is the global attractor conjecture concerning so-called "complex-balanced systems". Complex-balanced systems form a well-studied subclass of weakly reversible mass-action ODE systems that contain all so-called "detailed-balanced" systems and weakly reversible "deficiency zero" systems. Many properties about complex-balanced systems (as well as detailed-balanced systems and deficiency zero systems) were elucidated by Feinberg, Horn, and Jackson in the 1970s, and we provide only an overview here. (See any of the references $[9,12,13,17]$ for a definition of complex-balanced systems.)

For complex-balanced systems, it is known that there is a unique steady state within the interior of each invariant polyhedron $\mathcal{P}$. This steady state, called the Birch point in [9] due to the connection to Birch's theorem in algebraic statistics, has a strict Lyapunov function. Therefore local asymptotic stability relative to $\mathcal{P}$ is guaranteed $[13,17]$ (see Remark 6.7). An open question is whether all trajectories with an initial condition in the interior of $\mathcal{P}$ converge to the unique Birch point of $\mathcal{P}$. The assertion that the answer is "yes" is the content of the following conjecture, which was stated first by Horn in 1974 [16] and given the name "Global Attractor Conjecture" by Craciun et al. [9].

Conjecture 6.1 (global attractor conjecture). For any complex-balanced mass-action system and strictly positive initial condition $x^{0}$, the Birch point in $\mathcal{P}:=\left(x^{0}+H\right) \cap \mathbb{R}_{\geq 0}^{S}$ (see Definition 4.11) is a global attractor of the relative interior of the invariant polyhedron int $(\mathcal{P})$. 
In 1987, Feinberg [11] conjectured the following.

Conjecture 6.2 (Feinberg's persistence conjecture). For every confined, weakly reversible massaction ODE system $N$, the differential inclusion $\mathfrak{M}^{\ell}(N)$ is persistent.

Feinberg observed that the global attractor conjecture would follow from Conjecture 6.2, that is, persistence of weakly reversible reaction networks.

Our functoriality results reduce persistence to bounding the dynamics away from vertices of the hypercube - so it suffices to ensure that all species remain bounded away from 0 and $\infty$ in the positive orthant - at the price of considering tempered reaction systems. More precisely, we state the following two corollaries.

Corollary 6.3. Let $\mathcal{P}$ be a set of one or more of the following properties: integer, chemical, reversible, strongly connected, weakly reversible, endotactic, and strongly endotactic. Let $\mathcal{F}$ be the class of all confined reaction systems whose underlying reaction networks satisfy every property in $\mathcal{P}$. If the mass-action differential inclusions of all reaction systems in $\mathcal{F}$ are repelled by vertices after pushing forward to open hypercubes by a smooth order-preserving diffeomorphism, then they are repelled by the entire boundary.

Proof. Immediate from Corollaries 3.17 and 5.24.

The next result states that another approach to persistence is by proving that trajectories are bounded and that the origin is repelling; additionally, a more uniform such bound yields a permanence-like result.

Corollary 6.4. Let $\mathcal{P}$ be a set of one or more of the following properties: integer, chemical, reversible, strongly connected, weakly reversible, endotactic, and strongly endotactic. Let $\mathcal{F}$ be the class of all confined reaction systems whose underlying reaction networks satisfy every property in $\mathcal{P}$. If the mass-action differential inclusions of all reaction systems in $\mathcal{F}$ are repelled by the origin and every trajectory of such a differential inclusion is bounded, then

1) these differential inclusions are persistent; and

2) if $X$ is such a differential inclusion on $\mathbb{R}_{>0}^{S}$, and $K \subseteq \mathbb{R}_{>0}^{S}$ is a compact set for which there exists $A \in \mathbb{R}_{>0}$ such that every trajectory of $X$ that starts in $K$ remains bounded above by $A$ in each coordinate for all time, then for some compact set $K^{+} \subseteq \mathbb{R}_{>0}^{S}$, no trajectory of $X$ that begins in $K$ leaves $K^{+}$.

Proof. A differential inclusion on $\mathbb{R}_{>0}^{S}$ is repelled by the origin if and only if after pushing forward to open hypercubes the resulting differential inclusion is repelled by the origin: the open sets in the definition of repelled move between hypercubes and positive orthants via the diffeomorphism. Additionally, persistence of a mass-action differential inclusion is viewed with respect to the compactification $[0, \infty]^{S}$, so persistence is equivalent to persistence of the pushforward with respect to $[0,1]^{S}$. Finally, compact sets $K$ and bounds $A$ also move between hypercubes and positive orthants via the diffeomorphism. Thus, the conclusion follows from Corollaries 5.24, 3.18, and 3.21 (for which the repelled set is taken to be the origin - see also the description after Corollary 3.21).

Recently, Craciun, Nazarov, and Pantea [10] generalized Feinberg's persistence conjecture (Conjecture 6.2) in the following three ways: the weakly reversible hypothesis is weakened to endotactic, fixed reaction rate constants are allowed to vary within bounded intervals (i.e., a tempering), and the conclusion of persistence is strengthened to permanence $[10, \S 4]$.

Conjecture 6.5 (extended permanence conjecture). For every confined endotactic reaction system $N$, the differential inclusion $\mathfrak{M}^{\ell}(N)$ is permanent. 
Remark 6.6. Endotactic networks constitute a projective class (Corollary 5.24), and, in our view, it is this property that allowed Craciun, Nazarov, and Pantea to make their projectiontype arguments $[10,20]$. Similarly, the property of having only one linkage class is projective: a network with only one linkage class maintains this property after reduction. This type of argument was used in Anderson's proof of the global attractor conjecture for networks possessing only one linkage class [2]. Indeed, our work was motivated in part by the works both of Anderson and of Craciun, Nazarov, and Pantea.

Remark 6.7. Horn and Jackson [17] established that any complex-balanced mass-action ODE system $N$ on a set $S$ of species admits the strict Lyapunov function

$$
g_{\alpha}(x)=\sum_{i \in S} x_{i}\left(\log \frac{x_{i}}{\alpha_{i}}-1\right)
$$

where $\alpha \in \mathbb{R}_{>0}^{S}$ is a given complex-balanced steady state of $N$. Consequently, the differential inclusion $\mathfrak{M}^{\ell}(N)$ is repelled by the vertex 0 , and its trajectories are bounded away from faces not incident to 0 . The proof of this assertion, which is due in part to Anderson and Craciun et al. $[3,9]$, proceeds by showing that the Lyapunov function $g_{\alpha}$ has a local maximum at the vertex 0 as well as bounded level sets that do not allow trajectories to escape to infinity. If the class of complex-balanced systems were projective, then our arguments would have proved the global attractor conjecture. However, this is not the case: projections of complex-balanced systems need not be complex-balanced. Therefore, Theorem 5.23 does not apply: we can not prove that complex-balanced systems form a vertexical family. In fact, it can be shown that this family is not vertexical. On the other hand, weakly reversible or endotactic networks do define a vertexical family; recall Remark 6.6.

The motivation for our work was to make progress on Conjectures 6.1, 6.2, and 6.5. The next two theorems state what we have accomplished in this direction. First, we show that an extension of Feinberg's persistence conjecture in dimension $n$ implies the global attractor conjecture in dimension $n+1$, under an additional assumption that the origin is repelling.

Theorem 6.8. Let $n$ be a positive integer. If for every confined, weakly reversible reaction system $N$ with no more than $n$ species, both

1) $\mathfrak{M}^{\ell}(N)$ is persistent, and

2) $\mathfrak{M}^{\ell}(N)$ is repelled by the origin,

then the global attractor conjecture (Conjecture 6.1) holds for complex-balanced mass-action systems with $n+1$ or fewer species.

Proof. Let $\mathcal{X}$ denote the vertexical family of all differential inclusions that arise from endotactic networks. Let $N$ denote a confined, complex-balanced mass-action system with $|S| \leq n+1$. As mentioned after Conjecture 6.2, it suffices to prove that $N$ is persistent. By Remark 3.23 in the context of the empty repulsing index set, to prove that $N$ is persistent, it suffices to show that $(i) \mathfrak{M}^{\ell}(N)$ itself is persistent relative to all faces of $[0,1]^{S}$ that do not meet the origin and repelled by the origin of the hypercube, and $(i i)$ the lower-dimensional set $\mathcal{X}_{U}$ for each proper nonempty subset $U \subseteq S$ is persistent relative to all faces of $[0,1]^{U}$ that do not meet the origin and repelled by the origin of the hypercube $[0,1]^{U}$. Claim $(i)$ follows from the Lyapunov function, as explained in Remark 6.7: persistence relative to all faces that do not meet the origin is equivalent to having bounded trajectories. As for claim (ii), endotactic systems having lower dimension than $X$ are persistent by hypothesis, and repelled by the origin also by hypothesis. 
The next theorem reduces the global attractor conjecture and Feinberg's persistence conjecture to the following assertion that for mass-action differential inclusions arising from weakly reversible networks, trajectories are bounded and repelled by the origin.

Conjecture 6.9. For every confined, weakly reversible reaction system $N$, the differential inclusion $\mathfrak{M}(N)$ is repelled by the origin, and every trajectory of $\mathfrak{M}(N)$ is bounded.

Theorem 6.10. Conjecture 6.9 implies the global attractor conjecture (Conjecture 6.1).

Proof. Consider a confined weakly reversible reaction system $N$. By hypothesis, the differential inclusion $\mathfrak{M}(N)$ is repelled by the origin, and every trajectory of $\mathfrak{M}(N)$ is bounded, so $\mathfrak{M}(N)$ is persistent by Corollary 6.4.1. Thus, all weakly reversible systems are persistent, which implies the global attractor conjecture: see the discussion after Conjecture 6.2.

Conjectures 6.1, 6.2, 6.5, and 6.9 all remain open; for an overview of recent progress on these problems, we refer the reader to the work of Anderson $[3, \S 1.1]$. However, using the results presented in the current paper, we prove the following in a subsequent paper [15], which extends recent results of Anderson [2].

Theorem 6.11. For every confined strongly endotactic reaction system $N$, the differential inclusion $\mathfrak{M}^{\ell}(N)$ is permanent.

Proof. To be proved in a subsequent paper [15].

The key step contributed by the results in the current paper is Corollary 6.4 in the case of strongly endotactic networks. The approach in [15] shows that for the mass-action differential inclusions of strongly endotactic networks, outside a compact set the function $g_{\alpha}(x)$ in Remark 6.7 continues to behave like a Lyapunov function. Theorem 6.11 is established by an argument along the lines suggested in Remark 6.7.

\section{Acknowledgements}

MG was supported by a Ramanujan fellowship from the Department of Science and Technology, India, and, during a semester-long stay at Duke University, by the Duke MathBio RTG grant NSF DMS-0943760. EM had support from NSF grant DMS-1001437. AS was supported by an NSF postdoctoral fellowship DMS-1004380. The authors thank David F. Anderson, Gheorghe Craciun, and Casian Pantea for helpful discussions, and Duke University where many of the conversations occurred. The authors also thank the two referees, whose perceptive and insightful comments improved this work.

\section{References}

[1] Adleman L., Gopalkrishnan M., Huang M.D., Moisset P., Reishus D., On the mathematics of the law of mass action, arXiv:0810.1108.

[2] Anderson D.F., A proof of the global attractor conjecture in the single linkage class case, SIAM J. Appl. Math. 71 (2011), 1487-1508, arXiv:1101.0761.

[3] Anderson D.F., Global asymptotic stability for a class of nonlinear chemical equations, SIAM J. Appl. Math. 68 (2008), 1464-1476, arXiv:0708.0319.

[4] Anderson D.F., Shiu A., The dynamics of weakly reversible population processes near facets, SIAM J. Appl. Math. 70 (2010), 1840-1858, arXiv:0903.0901.

[5] Angeli D., De Leenheer P., Sontag E., A Petri net approach to persistence analysis in chemical reaction networks, in Biology and Control Theory: Current Challenges, Lect. Notes Contr. Inf., Vol. 357, Editors I. Queinnec, S. Tarbouriech, G. Garcia, S.I. Niculescu, Springer-Verlag, Berlin, 2007, 181-216, q-bio/0608019. 
[6] Angeli D., De Leenheer P., Sontag E.D., Persistence results for chemical reaction networks with timedependent kinetics and no global conservation laws, SIAM J. Appl. Math. 71 (2011), 128-146.

[7] Aubin J.P., Cellina A., Differential inclusions. Set-valued maps and viability theory, Grundlehren der Mathematischen Wissenschaften, Vol. 264, Springer-Verlag, Berlin, 1984.

[8] Banaji M., Mierczyński J., Global convergence in systems of differential equations arising from chemical reaction networks, J. Differential Equations 254 (2013), 1359-1374, arXiv:1205.1716.

[9] Craciun G., Dickenstein A., Shiu A., Sturmfels B., Toric dynamical systems, J. Symbolic Comput. 44 (2009), 1551-1565, arXiv:0708.3431.

[10] Craciun G., Nazarov F., Pantea C., Persistence and permanence of mass-action and power-law dynamical systems, SIAM J. Appl. Math. 73 (2013), 305-329, arXiv:1010.3050.

[11] Feinberg M., Chemical reaction network structure and the stability of complex isothermal reactors: I. The deficiency zero and deficiency one theorems, Chem. Eng. Sci. 42 (1987), 2229-2268.

[12] Feinberg M., Complex balancing in general kinetic systems, Arch. Rational Mech. Anal. 49 (1972), $187-194$.

[13] Feinberg M., Lectures on chemical reaction networks, unpublished lecture notes, 1979, available at http: //www. che.eng.ohio-state.edu/ FEINBERG/LecturesOnReactionNetworks/.

[14] Freedman H.I., Moson P., Persistence definitions and their connections, Proc. Amer. Math. Soc. 109 (1990), 1025-1033.

[15] Gopalkrishnan M., Miller E., Shiu A., A geometric approach to the global attractor conjecture, in preparation.

[16] Horn F., The dynamics of open reaction systems, in Mathematical Aspects of Chemical and Biochemical Problems and Quantum Chemistry (Proc. SIAM-AMS Sympos. Appl. Math., New York, 1974), SIAM-AMS Proceedings, Vol. 8, Amer. Math. Soc., Providence, R.I., 1974, 125-137.

[17] Horn F., Jackson R., General mass action kinetics, Arch. Rational Mech. Anal. 47 (1972), 81-116.

[18] Irwin M.C., Smooth dynamical systems, Pure and Applied Mathematics, Vol. 94, Academic Press Inc., New York, 1980.

[19] Lee J.M., Introduction to smooth manifolds, Graduate Texts in Mathematics, Vol. 218, Springer-Verlag, New York, 2003.

[20] Pantea C., On the persistence and global stability of mass-action systems, SIAM J. Math. Anal. 44 (2012), 1636-1673, arXiv:1103.0603.

[21] Savageau M.A., Biochemical systems analysis: I. Some mathematical properties of the rate law for the component enzymatic reactions, J. Theor. Biol. 25 (1969), 365-369.

[22] Siegel D., Johnston M.D., A stratum approach to global stability of complex balanced systems, Dyn. Syst. 26 (2011), 125-146, arXiv:1008.1622. 